\title{
1 Caenorhabditis elegans as an emerging model for studying the basic biology of
}

2 anorectic effects of nicotine

3 Robert Sobkowiak ${ }^{\mathrm{a} *}$, Piotr Kaczmarek ${ }^{\mathrm{b}}$, Mateusz Kowalski ${ }^{\mathrm{b}}$, Rafał Kabaciński ${ }^{\mathrm{b}}$, Andrzej

4 Lesicki $^{\mathrm{a}}$

5

$6{ }^{\text {a }}$ Department of Cell Biology, Adam Mickiewicz University, Faculty of Biology

7 ul. Umultowska 89, 61-614 Poznań, Poland

$8{ }^{\mathrm{b}}$ Poznan University of Technology, Faculty of Electrical Engineering, Institute of Control and

9 Information Engineering, ul. Piotrowo 3a, 60-965 Poznań, Poland

10

11 * Corresponding author; tel.: +48 61 8295822; fax: +48 61 8295829;

12 e-mail: robsob@amu.edu.pl

15 Funding: This study was supported by funds from the Department of Cell Biology, Faculty of 16 Biology, Adam Mickiewicz University (Poznań, Poland).

17

18 Competing interests: The authors have declared that no competing interests exist. 


\section{Abstract}

21 Nicotine decreases food intake, and smokers often report that they smoke to control 22 their weight. To see whether similar phenomena could be observed in the model organism 23 Caenorhabditis elegans, we challenged drug-naïve nematodes with a chronic low (0.01 mM) 24 and high $(1 \mathrm{mM})$ nicotine concentration for $55 \mathrm{~h}$ (from hatching to adulthood). After that, we 25 recorded changes in their behavior in a nicotine gradient, where they could choose a desired 26 nicotine concentration. By using a combination of behavioral and morphometric methods, we 27 found that both nicotine and food modulate worm behavior. In the presence of food the 28 nematodes adapted to the low nicotine concentration, when placed in the gradient, chose a 29 similar nicotine concentration like $C$. elegans adapted to the high nicotine concentration. 30 However, in the absence of food, the nematodes adapted to the low nicotine concentration, 31 when placed in the gradient of this alkaloid, chose a similar nicotine concentration like naïve 32 worms. The nematodes growing up in the presence of high concentrations of nicotine had a 33 statistically smaller body size, compared to the control condition, and the presence of food did 34 not cause any enhanced slowing movement. These results provide a platform for more 35 detailed molecular and cellular studies of nicotine addiction and food intake in this model 36 organism.

39 Keywords: Nicotine gradient; Nicotine exposure; Behavior; Body size; Food intake; 40 Caenorhabditis elegans. 


\section{Introduction}

Tobacco smoking is the largest single preventable cause of many chronic diseases and death (WHO, 2012). Most of the tobacco cigarette's toxicity is related to the combustion process. Although tobacco smoking has declined since the 1950s, the use of e-cigarettes has increased, attracting both former tobacco smokers and never smokers. Regardless of nicotine source, method for delivering, and other concomitant substances, nicotine is the most biologically active ingredient of seriously harmful tobacco smoke and the potentially harmful e-cigarette aerosol (Grana et al., 2014; Schuller, 2009; Sobkowiak and Lesicki, 2011).

Some smokers report that they smoke as a method of weight control (Nichter et al., 2004). Indeed, smokers have a notably lower body mass index than nonsmokers and gain weight when they quit (Filozof et al., 2004; Jo et al., 2002; Mineur et al., 2011). These effects on body weight have been attributed to nicotine in tobacco, because nicotine decreases food intake in animal models. In humans, nicotine has some effects on peripheral energy metabolism (Filozof et al., 2004; Jo et al., 2002) and this was also observed in the model nematode Caenorhabditis elegans (Sobkowiak et al., 2016).

Nicotine competes with acetylcholine for binding to specific membrane receptors, socalled nicotinic cholinergic receptors (nAChRs). They are widely expressed in the nervous system and skeletal muscle. Nicotinic receptors are also present in many cell types, e.g. epithelial, blood, fat, and cancer cells (Liu et al., 2004; Sobkowiak and Lesicki, 2011). Also nicotine metabolites have significant effects on the body (Benowitz et al., 2009; Sobkowiak and Lesicki, 2013). Early nicotine exposure has been associated with many long-term consequences that include neuroanatomical alterations as well as behavioral and cognitive deficits (Rose et al., 2013). Moreover, nicotine is a highly addictive substance with negative effects on animal and human brain development, which is still ongoing in adolescence (Benowitz, 2010; Grana et al., 2014).

Locomotion reflects the integration of many aspects of both the environment and the internal state of the worm, and therefore can be a sensitive measure of behavioral state after nicotine application. As an animal travels through its environment, its nervous system detects sensory cues, evaluates them based on context and the experience of the animal, and converts the information into adaptive movement. $C$. elegans has behavioral states that are evident as long-term locomotor patterns that differ in well-fed or starved animals. When feeding on a bacterial lawn, C. elegans switches between 2 behavioral patterns. It spends $80 \%$ of its time dwelling, i.e. feeding on bacteria while moving slowly and staying in a restricted area. At rare intervals, however, it switches into an alternative behavioral state called roaming, which 
76 involves rapid locomotion across the lawn. When removed from the bacterial lawn, $C$.

77 elegans switches to a behavioral state in which it moves rapidly and reverses frequently. This

78 strategy is called pivoting, area-restricted search, or local search, and has the same basic runand-pirouette components as directed chemotaxis. Over the next 30 min, reversals are suppressed, shifting the animals to another behavioral state, called traveling or dispersal (Bargmann, 2006 and the papers cited therein).

82 Worms navigate to favorable conditions by chemotaxis and aerotaxis (Gray et al., 83 2005). C. elegans shows chemotaxis to various odorants and water-soluble chemoattractants, 84 like nicotine, by sensing them mainly with sensory neurons whose sensory endings are 85 located in amphid sensory organs at the anterior tip of the animal. The strategy of chemotaxis 86 in this organism was previously studied by Pierce-Shimomura et al. (1999), who found the 87 "pirouette strategy" for chemotaxis to water-soluble chemoattractants. Iino and Yoshida (2009) reported the discovery of a second mechanism for chemotaxis, called the weathervane mechanism. In that strategy, the animals respond to a spatial gradient of the chemoattractant and gradually curve toward a higher concentration of the chemical.

Caenorhabditis elegans is a powerful genetic model for studying various questions in 92 neurobiology, including nicotine dependence. For example, it has been reported that $C$. 93 elegans responds to nicotine in both a concentration-dependent and a time-dependent manner, 94 and shows a wide range of behavioral responses to nicotine (Feng et al., 2006; Sobkowiak et 95 al., 2011). These include a continuous higher locomotion speed (acute response), tolerance, withdrawal, and sensitization via nAChR (Feng et al., 2006). Dual effects of nicotine on locomotion speed, which are dependent on differences in its dosage and treatment duration, have also been revealed (Sobkowiak et al., 2011). Moreover, C. elegans is capable of navigating up a nicotine gradient (Sellings et al., 2013).

100 In the current study we observed spontaneous behavior in the presence or absence of

101 food as well as a nicotine gradient to describe the effects of low and high nicotine 102 concentration exposure during larval development in adult C. elegans. 


\section{Results}

\section{$105 \quad 2.1 \quad$ Body size of worms}

106 Nicotine affected the body size of nematodes. Worms challenged from hatching to 107 adulthood $\left(55 \mathrm{~h}, 22^{\circ} \mathrm{C}\right)$ with $1 \mathrm{mM}$ nicotine were smaller than in control conditions (Fig. 3).

108 The worms adapted to the high nicotine concentration $(1 \mathrm{mM})$ were about $5 \%$ shorter (Fig. 109 3A), with about 12\% smaller surface (Fig. 3B), and about 22\% smaller body volume, as 110 compared to control nematodes (Fig. 3C). The nematodes adapted to a low nicotine 111 concentration $(0.01 \mathrm{mM})$ were on average about $4.5 \%$ longer (Fig. 3a), with about 5.5\% 112 smaller volume (Fig. 3C), as compared to control nematodes. However, there was no 113 statistically significant difference between these 2 groups.

\section{$114 \quad 2.2 \quad$ Average values of factors describing worm behavior}

\section{$115 \quad$ 2.2.1 Average speed of movement}

116 The average speed of movement of well-fed C. elegans worms (Fig. 4A) was the fastest 117 in the absence of food (F-nA0), and their locomotion slowed down when food was present $118(\mathrm{~F}+)$. A similar effect of slowing the movement, but also in the absence of food, was evoked 119 by the gradient of nicotine $(\mathrm{F}-\mathrm{N})$.

120 In the presence of food on the nicotine-free Petri dish, there was no statistically 121 significant difference in locomotion speed between the nematodes having the first contact 122 with this alkaloid (naïve worms) and nematodes adapted to $0.01 \mathrm{mM}$ nicotine (Fig. 4A, $123 \mathrm{~F}+\mathrm{nA} 0$ vs. F+nA0.01). In the presence of food and without nicotine in the center of the assay 124 plate, the worms adapted to $0 \mathrm{mM}$ nicotine $(\mathrm{F}+\mathrm{nA} 0)$ and those adapted to $0.01 \mathrm{mM}$ nicotine 125 (F+nA0.01) moved with the same speed (no significant differences, Fig. 4A).

126 The worms adapted to $1 \mathrm{mM}$ nicotine in the presence of nicotine gradient moved with the 127 same speed independently of the presence or absence of food (Fig. 4A, no significant 128 differences between F+NA1 and F-NA1). It is noteworthy that the nematodes adapted to $1291 \mathrm{mM}$ nicotine $(\mathrm{F}+\mathrm{nA} 1)$ in the presence of food unexpectedly moved farthest from the start 130 position and the presence of food appeared to stimulate the process. 


\subsubsection{Average distance from the start position}

132 Nematodes moved away from the starting point in a range of $3 \mathrm{~mm}(\mathrm{~F}+\mathrm{KA} 0)$ to $18 \mathrm{~mm}$ 133 (F-NA0) (Fig. 4B). The presence of food in the absence of nicotine gradient evoked in naïve 134 worms a tendency to keep close to the start position (Fig. 4B, F-nA0 vs. F+nA0). Median 135 value of the distance from the starting point for the worms adapted to $0.01 \mathrm{mM}$ nicotine was 136 almost the same but the range of $25 \%-75 \%$ narrowed (Fig. 4B, F-nA0.01 vs. F+nA0.01).

In the presence of food we observed a linear tendency with a significantly $(p<0.05$,

138 Kruskal-Wallis ANOVA by ranks test) increased distance of worms from the starting point 139 (Fig. 4B, F+nA0 lowest value, F+nA0.01, F+nA1 highest values).

140 We observed an opposite tendency when a gradient of nicotine appeared on the plate. 141 Independently of the presence or absence of food, nicotine extorted a decrease in the distance 142 from the start position in line with the increase in nicotine concentration to which the worms 143 were earlier adapted (Fig. 4B, F+NA0, F+NA0.01, F+NA1). Interestingly, we revealed no 144 statistical difference in average distance from the start position between the worms adapted to $1451 \mathrm{mM}$ nicotine in the presence of food and absence of food (Fig. 4B, F+NA1 vs. F-NA1)

\subsubsection{Average distance from the peak}

147 The peak was the central point where nicotine or water was applied on the gradient assay 148 plate. The radius in which nematodes were present, measured from the peak to worm location, 149 was in the range from about $4 \mathrm{~mm}(\mathrm{~F}-\mathrm{NA} 0$, F-NA0.01) to about $21 \mathrm{~mm}(\mathrm{~F}+\mathrm{nA}$ 0.01) (median 150 value, Fig. 4C). When there was no nicotine in the center of the gradient assay plate, the 151 shortest distance from the peak was observed for the worms adapted to $1 \mathrm{mM}$ nicotine $152(\mathrm{~F}+\mathrm{nA} 1)$. When the gradient of nicotine appeared and in the absence of food, the worms 153 adapted to $0 \mathrm{mM}$ and $0.01 \mathrm{mM}$ reached the peak and behaved in the same way (Fig. 4C F154 NA0, F-NA0.01, no statistical difference). The worms adapted to $0.01 \mathrm{mM}$ nicotine in the 155 presence of food were not attracted to the peak and they chose food and kept distance like on 156 the plates without nicotine (Fig. 4C, compare median values for F+nA0.01 vs. F+NA0.01 vs. 157 F-NA0.01).

\subsubsection{Average nicotine concentration preferred by worms}

159 In our experiment, nematodes could select the concentration of nicotine in which they 160 wanted to stay. Their movements depended on the presence of food and on adaptation to 
161 nicotine. In the absence of food, worms preferred on gradient assay plates higher

162 concentrations of nicotine in comparison to the worms placed on plates without food (Fig.

163 4D). The nematodes adapted to the low concentration of nicotine $(0.01 \mathrm{mM})$ behaved

164 differently in comparison to the nematodes adapted to the high concentration of nicotine (1

$165 \mathrm{mM}$ ). The worms adapted to $1 \mathrm{mM}$ chose the lowest nicotine concentration independently of

166 the presence or absence of food (Fig. 4D, F+NA1 and F-NA1). In the presence of food the

167 worms adapted to $0.01 \mathrm{mM}$ nicotine behaved like the worms adapted to $1 \mathrm{mM}$ nicotine (Fig.

168 4D, F+A0.01 vs. F+NA1, no statistical difference). However, in the absence of food, the 169 worms adapted to $0.01 \mathrm{mM}$ nicotine behaved like the worms adapted to $0 \mathrm{mM}$ nicotine (Fig. 170 4D, F-NA0 vs. F-A0.01, no statistical difference) and chose a very high concentration of 171 nicotine.

\section{$172 \quad 2.3 \quad$ Time-dependent response of $C$. elegans to the nicotine gradient}

\section{$173 \quad$ 2.3.1 Time-dependent speed of movement}

174 The speed of the worms varied with time (Fig. 5). In most cases, we observed a 175 decreasing trend in the speed of their movements, and its frequent oscillations. In control 176 conditions, i.e. in the absence of a gradient of nicotine and food (F-nA0), we observed 177 oscillations of the speed, while the nematodes in variant F-nA0.01 show a downward trend in 178 the speed of movement (Fig. 5E). The long period of reduced locomotion activity was 179 observed particularly in control nematodes (Fig.Fig. 5b, time 500-1100 s) and those adapted 180 to $0.01 \mathrm{mM}$ nicotine (Fig. 5E, time 2500-3600 s; Fig. 5G, time 700-1400 s).

\subsubsection{Time-dependent changes in distance from the start position}

182 Fig. 6 shows the variation in the distance from the start position of worms (measured 183 from the start position to the actual worm location). Generally during the experiment the 184 nematodes moved away from the starting point. In some experimental conditions, nematodes 185 remained at a relatively stable distance from the start position (Fig. 6B,F,K). This applied 186 especially to the worms that never had contact with nicotine before, and were transferred to a 187 Petri dish with bacteria and no nicotine gradient (Fig. 6C, F+nA0). Those worms tended to 188 move away from the starting point about $1200 \mathrm{~s}$ after application (.Fig. 6C). Farthest mean 189 distance from the start position was recorded for the nematodes adapted to $0.01 \mathrm{mM}$ nicotine, 190 on the plate without nicotine and bacteria (variant F-nA0.01, Fig. 6E). 


\subsubsection{Time-dependent changes in distance from the peak}

192 The distance between worms and the peak varied with time (Fig. 7), but was

193 exceptionally constant for the nematodes adapted to $1 \mathrm{mM}$ nicotine and placed on Petri dishes

194 with food and nicotine gradient (F+NA1, Fig. 7I). Interesting behavior of nematodes was 195 observed in variants F-NA0 and F-NA0.01 .Fig. 7B,F), where almost immediately after 196 application they moved towards the central peak of nicotine.

\section{$197 \quad$ 2.3.4 Time-dependent changes in preferred concentration of nicotine}

198 In a radial gradient of nicotine, control wild-type nematodes and those adapted to 0.01 $199 \mathrm{mM}$ nicotine usually reached the gradient peak by moving almost directly up the gradient 200 (Fig. 8A,C, F-NA0 and F-NA0.01). The preference for higher concentrations appeared about $201300 \mathrm{~s}$ after the application of nicotine in the center of the Petri plate. However, the nematodes 202 adapted to $0.01 \mathrm{mM}$ nicotine, when placed on plates with food, avoided nicotine for the first $203900 \mathrm{~s}$ of experiment (Fig. 8D, time 0-1000s). Nematodes adapted to $1 \mathrm{mM}$ nicotine avoided 204 nicotine for the first $1500 \mathrm{~s}$ of the experiment (Fig. 8E, f, time 0-1000s). 
206

207

208

209

210

211

212

213

214

215

216

217

218

219

220

221

222

223

224

225

226

227

228

229

230

231

232

233

234

235

236

237

238

239

\section{Discussion}

In the experiments we used 3 groups of nematodes: naïve worms (which never had contact with nicotine before), worms adapted to a low uniform nicotine concentration (0.01 $\mathrm{mM}$ ) and to a high uniform nicotine concentration (1 mM) (Fig. 1B). The adaptation to nicotine lasted from hatching to adulthood, and next we investigated the behavior of these nematodes in a nicotine gradient (Fig. 1C). Nicotine was administered in the center of the Petri plate in one bolus dose. Theoretically, the bolus of $1 \mu \mathrm{L}$ of $580 \mathrm{mM}$ nicotine diluted in $5.8 \mathrm{~mL}$ of medium in Petri dish diffuses to final $0.1 \mathrm{mM}$ nicotine concentration and disappearing of nicotine gradient after about $12 \mathrm{~h}$. However, we started our experiments immediately after administration of nicotine onto the Petri dish. In our previous study, in uniform nicotine concentration, within the first $3600 \mathrm{~s}$ we observed the largest difference in the behavior of control naïve nematodes and those in the presence of $0.1 \mathrm{mM}$ nicotine (Sobkowiak et al., 2011). In this study, we used a 10-fold lower (0.01 mM) and 10-fold higher nicotine concentration $(1 \mathrm{mM})$ during adaptation of worms. These 2 concentrations were considered "effective dosages", as our previous report revealed that dosages in this range cause changes in locomotion (Sobkowiak et al., 2011, see Fig. 2 in that article).

We performed chemotaxis assays by tracking individual worms in the radial Gaussianshaped gradients of nicotine. C. elegans has a highly developed chemosensory system that enables it to detect a wide variety of volatile (olfactory) and water-soluble (gustatory) cues associated with food and chemicals (Bargmann, 2006). During the experiments, the nematodes were allowed to move freely around the Petri dishes, and able to select the desired nicotine concentration. Sellings et al. (2013) suggest that nicotine acts as a rewarding substance in $C$. elegans. The nematodes approach a point source of nicotine in a timedependent and concentration-dependent manner. Those authors revealed that wild-type worms climb the nicotine gradient, and suggested that worms exposed to $50 \mathrm{mM}$ nicotine can approach appetitive stimuli, once removed from the nicotine source. This is in line with our result, with the exception of the high nicotine concentration experimental variant. The naïve animals and worms adapted to low nicotine concentration reached the peak of the gradient, defined as a circular region with a radius of $5 \mathrm{~mm}$ located at the center of the plate (Fig. 2A, Fig. 7B, F), and were trapped in the nicotine area when the nicotine concentration exceeded $500 \mathrm{mM}$ at the beginning of experiment (Fig. 8A,B,C). The cuticle of C. elegans is a significant barrier for drug permeability, thus the internal concentrations in the worm's body fluid is likely to be substantially lower than the dosing medium concentration (Wolf and Heberlein, 2003). However, high concentrations of nicotine induce muscle hypercontraction 
240 paralysis (Matsuura et al., 2013; Sobkowiak et al., 2011) and cause rapid transient paralysis of

241 body wall muscles, manifested in an extremely low speed of locomotion (Fig. 5A, time 300-

242 900s; Fig. 5F, 1200-1600s). This suggests that the nematodes were affected by nicotine

243 toxicity and paralysis.

244 The naïve animals and those adapted to the low nicotine concentration tested in the 245 nicotine gradient were significantly more unlikely to reach the center when on the Petri dish 246 the food was in a uniform concentration (Fig. 5C and $8 \mathrm{D}, \mathrm{H}$ ). There were no statistically 247 significant differences in distance from the peak between the naïve animals and the worms 248 adapted to the low nicotine concentration (Fig. 4C, F-NA0 vs. F-NA0.01).

Due to the nature of the behavior of the nematode C. elegans, the distribution of all the measured values was not normal. Therefore, for this type of distribution, an appropriate measure of central tendency is the median. Nonparametric tests more easily demonstrate the existence of a significant statistical difference, especially when a large amount of data is available. In our study, a single experiment provides 7200 data, because records were taken every $0.5 \mathrm{~s}$ during the $3600 \mathrm{~s}$ of the experiment. Additionally, the experiments were repeated 5 times, to increase the pool of results. Thus most of the experimental variants differed significantly from one other. In our view, more biologically important are the results which do not differ in a statistically significant way, despite the distinctly different experimental conditions.

Caenorhabditis elegans chemotaxes to bacteria, its natural food source, by following both water-soluble and volatile cues. Because of this difference in sensitivity, and because the diffusion of small molecules through air is much more rapid than through water, it is likely that volatile odors are used first for long-range chemotaxis, and later water-soluble attractants are used for short-range chemotaxis (Bargmann, 2006). Many studies have shown that the absence or presence of food markedly influences the average speed of wild-type worms (de Bono and Bargmann, 1998; Ramot et al., 2008). Sawin et al. (2000) reported that the feeding status (well-fed or starved) as well as the presence or absence of food (bacteria) affects the rate of locomotion of $C$. elegans. Sawin et al. (2000) reported a decrease in the locomotory rates of well-fed adult $C$. elegans on a bacterial lawn (food) compared with those on plates lacking bacteria, and defined this response as the "basal slowing response". Nicotine exposure 270 alters behaviors in C. elegans, including pharyngeal pumping, which disturbs nutrition (Matta 271 et al., 2007). Pharynges that have been dissected from wild-type worms hypercontract in 0.1 $272 \mathrm{mM}$ nicotine (McKay et al., 2004). Nematodes in the presence of high concentrations of 273 nicotine have smaller body size (Fig. 3) probably because they eat less, as compared to 
274 control nematodes, thereby releasing less serotonin, and do not show enhanced slowing on

275 food (Fig. 5A and 6K,L). The neurosecretory-motor neurons that have sensory endings in the

276 lumen of the pharynx (which thus might sense food) also synthesized serotonin (Chase and

277 Koelle, 2007). Serotonin is required for the so-called "enhanced slowing response" (Sawin et

278 al., 2000). C. elegans individuals adapted to the high nicotine concentration were smaller than

279 those in the control, probably because in the presence of food their serotonergic neurons did

280 not release an increased amount of serotonin, which did not inhibit the motor circuit to a

281 greater extent than in the basal slowing response to effect the enhanced slowing response (Fig.

282 4A F+nA0 vs. F+nA1). Without such serotonin release, the enhanced slowing response could

283 not occur. Thus on the plate with food, the worms adapted to the high nicotine concentration

284 moved faster than the control worms. The animals slow their locomotion rate dramatically

285 when they encounter a bacterial lawn (Fig. 5A and 6C,D,G,H) and, as mentioned above, the

286 enhanced slowing response requires serotonin (Sawin et al., 2000). Serotonin signaling thus

287 provides a mechanism to ensure that animals absolutely do not leave a food source once they

288 have encountered it (Chase and Koelle, 2007). That is probably why we observed a very short

289 distance from the start position for the control worms and a little larger distance for those

290 adapted to the low concentration to nicotine (Fig. 6C,G).

291 Sellings et al. (2013) were the first to reveal that nicotine serves as a primary motivating

292 stimulus in C. elegans. Their research suggests that dopamine (DA) transmission is important

293 in mediating the nicotine-motivated behaviors (Sellings et al., 2013). The presence of bacteria

294 is perceived by mechanosensory stimulation via 8 dopaminergic sensory neurons (Sawin et

295 al., 2000). The level of dopamine is higher in the presence of nicotine. We hypothesized that

296 in nematodes adapted to high nicotine concentration $(1 \mathrm{mM})$, the alkaloid mimics bacterial

297 mechanosensory stimulation via sensors of dopaminergic neurons, thus nematodes eat less

298 and have smaller body size (Fig. 3A, F-nA0 vs. F-nA1). The dopamine signaling in well-fed

299 animals encourages the animal to stay in the proximity of food but still may permit limited

300 exploration for new or better food sources (Chase and Koelle, 2007). This is a likely

301 explanation why the presence of nicotine encouraged nematodes to move away from the

302 application site on the Petri dish (Fig. 6D,H).

303 The effects of nicotine on locomotion vary according to dose and over time

304 (Sobkowiak et al., 2011). In other animal models, acute administration of nicotine evokes also

305 dual changes in locomotor activity (Matta et al., 2007). Innate chemosensory preferences are

306 often encoded by sensory neurons that are specialized for attractive or avoidance behaviors.

307 Tsunozaki et al. (2008) show that one olfactory neuron in Caenorhabditis elegans, AWC ${ }^{\mathrm{ON}}$, 
308 has the potential to direct both attraction and repulsion. Attraction, the typical AWC ${ }^{\mathrm{ON}}$

309 behavior, requires a receptor-like guanylate cyclase GCY-28 that acts in adults and localizes

310 to $\mathrm{AWC}^{\mathrm{ON}}$ axons (Tsunozaki et al., 2008). Interestingly, in our previous research we found

311 GCY-28 only in naïve worms, which were distinctively attracted by nicotine. GCY-28 was

312 absent in the protein complexes involved in response to low and high concentration of

313 nicotine (Sobkowiak et al., 2016). This may suggest that in the presence of nicotine we

314 observed the same kind of switching by presence or absence of food in control nematodes and

315 worms adapted to low nicotine concentration (Fig. 4D).

316 Nicotine induces profound behavioral responses in C. elegans that mimic those

317 observed in mammals. The genes and pathways regulating nicotine dependence in mammals

318 are functionally conserved in C. elegans, including nicotinic acetylcholine receptors

319 (nAChRs, the molecular target of nicotine) and serotonin and dopamine-mediated

320 neurotransmission. In this study, the exposure started from hatching and lasted $55 \mathrm{~h}$, i.e. at

321 least 3/4 of the C. elegans life cycle. Sellings et al. (2013) demonstrated, and we confirmed

322 this finding, that $C$. elegans exhibits motivational behavior patterns towards nicotine, similar

323 to those observed in mammalian models. The motivational behavior pattern is modulated, as

324 we show, by food.

325 We revealed that nicotine can reduce worm body size (Fig. 3). The anorectic effects of

326 smoking have been well documented in human subjects, and the principal reason cited by

327 female teenagers for why they smoke is weight control. On average, smokers weigh $5 \mathrm{~kg}$ less

328 than nonsmokers and have significantly lower body mass index than nonsmokers. Similarly,

329 nicotine decreases feeding in animal models, suggesting that nicotine in tobacco is important

330 for the effects of smoking on appetite (Picciotto and Kenny, 2013).

331 Our results confirm that $C$. elegans may serve as a useful model organism for nicotine-

332 motivated and food-motivated behaviors that could help in understanding the mechanisms

333 underlying the anorectic effects of taking nicotine. Thus research on C. elegans may facilitate

334 the development of novel treatments to help with smoking cessation and with preventing or

335 treating obesity.

336

337

338

339

340 


\section{Materials and methods}

\section{$342 \quad 4.1 \quad$ Caenorhabditis elegans maintenance}

343 All tests were performed on the wild-type Bristol N2 strain of C. elegans obtained from 344 the Caenorhabditis Genetics Center (CGC) at the University of Minnesota (Duluth, 345 Minnesota, USA). Standard methods were used for the maintenance and manipulation of 346 strains (Stiernagle, 2006). Nematodes were kept at $22^{\circ} \mathrm{C}$ on nematode growth medium (NGM)

347 agar plates seeded with Escherichia coli strain OP50 as a source of food according to a 348 standard protocol (Brenner, 1974). Chunks of mixed-stage starved worms were transferred 349 onto enriched nematode growth (ENG) plates (50 $\mathrm{mm}$ in diameter) seeded with bacterial food 350 (E coli OP50), and the worms were allowed to grow for about 5 days at $22^{\circ} \mathrm{C}$ (Fig. $1 \mathrm{~A}$ ).

\section{$352 \quad 4.2$ Chronic exposure of $C$. elegans to uniform nicotine concentration}

(-)-Nicotine (free base) was obtained from Sigma-Aldrich. The drug was added directly to the NGM medium and allowed to diffuse throughout the medium for $48-72 \mathrm{~h}$ to ensure a uniform concentration.

To minimize interaction between $E$. coli and nicotine, 10 drops of liquid culture of $E$. coli OP50 were added just before chronic exposure of $C$. elegans to nicotine on agar plates.

358 Bacteria were evenly distributed by a spreader on the surface of the NGM medium, and the

359 plates with the lids removed were left to dry for about $10 \mathrm{~min}$. The short drying of the agar 360 surface enabled the nematodes free movement and proper nutrition.

361 When the culture of nematodes contained mostly adults able to lay eggs, chunks of the 362 agar with nematodes were transferred onto the plates for chronic exposure to a uniform 363 nicotine concentration $(0.01 \mathrm{M}$ or $1 \mathrm{mM}$, Fig. 1B). In the controls, the chunks were 364 transferred to nicotine-free NGM plates. To synchronize the worms, parent worms were 365 allowed to lay eggs for $3 \mathrm{~h}$ and next the adults were removed. The remaining eggs were 366 incubated for $55 \mathrm{~h}$ at $22^{\circ} \mathrm{C}$ to adulthood (Altun and Hall, 2009). The dosages were selected 367 based on our preliminary study as well previous reports (Feng et al., 2006; Matta et al., 2007; 368 Sobkowiak et al., 2011; Waggoner et al., 2000), in which nicotine treatment had a biphasic 369 response. 


\subsection{Measurement of worm size}

371

372

373

374

375

376

377

378

379

380

381

382

383

384

385

386

387

388

389

390

391

392

393

394

395

396

397

398

399

400

401

The measurements of worm size and the behavioral nicotine gradient experiments were performed using adult hermaphrodites, which were kept in the presence of nicotine from hatching to adulthood. Body size of adult worms was estimated in control conditions $(0 \mathrm{mM}$ nicotine) and after $55 \mathrm{~h}$ exposure to 0.01 and $1 \mathrm{mM}$ nicotine. To measure body length, volume, and surface area, the worms were put on an NGM plate, and pictures were taken using a stereomicroscope and analyzed using WormSizer, as previously described (Moore et al., 2013). WormSizer is open-source software that is useful for detecting relatively subtle phenotypes and morphological changes that may have been difficult to assess upon visual inspection. Eight biological replicates, typically including 12 individuals per experimental variant, were analyzed.

\subsection{Behavioral nicotine gradient assay}

In all the experiments we used young adult worms exposed to $0 \mathrm{mM}$ (control), 0.01 $\mathrm{mM}$, and $1 \mathrm{mM}$ nicotine. After $55 \mathrm{~h}$ of nicotine exposure, single young adult hermaphrodites from each adaptation variant were transferred to assay plates containing NGM medium with 4 treatment variants (Fig. 1C):

(1) no food, no radial Gaussian-shaped nicotine gradient (F-n);

(2) no food, a radial Gaussian-shaped nicotine gradient (F-N);

(3) spatially uniform concentration of food, no radial Gaussian-shaped nicotine gradient $(\mathrm{F}+\mathrm{n})$;

(4) spatially uniform concentration of food, a radial Gaussian-shaped nicotine gradient $(\mathrm{F}+\mathrm{N})$.

The behavioral nicotine gradient assays were performed in standard Petri dishes (inner diameter $92 \mathrm{~mm}$ ) containing $5.8 \mathrm{~mL}$ of NGM. In variants 3 and 4, NGM agar was seeded with 3 drops of the same medium with E. coli OP50, while in variants 1 and 2 the plates were seeded with 3 drops of sterile medium without E. coli (i.e. without food), and allowed to desiccate for about $10 \mathrm{~min}$. The radial Gaussian-shaped nicotine gradient was formed by adding $1 \mu \mathrm{L}$ of $580 \mathrm{mM}$ nicotine solution in water at the center of the plate just before the tracking. Nicotine concentration was estimated in the range of $0-580 \mathrm{mM}$, according to the diffusion equation for a point bolus in a thin slab (Crank, 1975) (Fig. 2B).

For each assay plate, time was recorded for estimation of nicotine concentration during the assay. At each time point of the assay, the concentration of nicotine (mM) at the position of the worm was estimated according to the solution of the diffusion equation (Crank, 1975) 
402 for a point bolus in a cylindrical, aqueous volume having the same dimensions as the agar in

403 the assay plate (diameter $9.2 \mathrm{~cm}$; depth $0.1 \mathrm{~cm}$ ). Exact nicotine concentration (in $\mathrm{mM}$ ) was

404 calculated by the solution of Fick's equation for 2-dimensional diffusion with no border

405 (Crank, 1975; lino and Yoshida, 2009).

406

$$
C=\frac{N_{0} e^{-\frac{r^{2}}{400 D t}}}{4 \Pi d D t}
$$

407 The Fick's equation for 2-dimensional diffusion with no border: $C=$ nicotine concentration

$408[\mathrm{mM}] ; N_{0}=0.58$ [mmole] is the number of moles of nicotine spotted $(1 \mu \mathrm{L}$ of $580 \mathrm{mM}$

409 nicotine contains 0.58 mmoles of nicotine); $D=0.00000042\left[\mathrm{~cm}^{2} / \mathrm{s}\right]$ is the diffusion

410 coefficient of nicotine (estimated basing on Sellings et al., 2013); $d=0.1[\mathrm{~cm}]$ is the depth of

411 the agar; $t=$ is the time [s] after spotting nicotine at the center of the plate; $r=$ is the distance

$412[\mathrm{~cm}]$ between the peak of the gradient and the location of the animal (Crank, 1975; Iino and

413 Yoshida, 2009).

414

To study the behavior of worms, we used an automated tracking system to follow 416 individual young adults crawling on NGM plates (Kowalski et al., 2014). Single young adults 417 (aged $\sim 55 \mathrm{~h}$ ) were manually picked off an adaptation plate (Fig. 1B) and placed using a 418 platinum pick in a $1-\mu \mathrm{L}$ droplet of water on the agar surface of the gradient assay plate (Fig. 419 1C), $15 \mathrm{~mm}$ from the plate center (Fig. 2A). Putting a worm in a droplet of water is an 420 effective method for rapid transferring of a single animal without scratching the agar surface 421 (important for obtaining high-contrast videos and perfect tracking) and for testing the 422 condition of the worm (injured nematodes, which could not properly swim in a drop of water, 423 were rejected). The surface tension of the drop of water prevented the nematode from 424 creeping out. Next, the plate was placed in a device for tracking nematodes. Observation of 425 the nematode allowed us to notice the moment when the $1-\mu \mathrm{L}$ drop disappeared by 426 evaporation/absorption in agar and released the worm, which could then freely move on the 427 plate. At that moment, in the center of the plate, $1 \mu \mathrm{L}$ of $580 \mathrm{mM}$ nicotine or $1 \mu \mathrm{L}$ of $\mathrm{H}_{2} \mathrm{O}$ was 428 spotted (Fig. 1C and 2A). Worm tracking began no more than $10 \mathrm{~s}$ after the application of nicotine or water in the center of the plate. Each worm was tracked for $3600 \mathrm{~s}$ or until it reached the edge of the plate. 


\subsection{Worm tracking system}

432

433

434

435

436

437

438

439

440

441

442

443

444

445

446

447

448

449

450

451

452

Custom worm tracker software (WormSpy) was used to move the camera automatically to re-center the worm under the field of view during recording (Kowalski et al., 2014). The automated tracking system comprises a stereomicroscope (Olympus SZ11), a modified (with unscrewed lens) web camera (Logitech QuickCam Pro 9000) with $640 \times 480$ resolution to acquire worm videos, and a desktop PC running under Windows 7 . The tracking system located the worm's centroid (defined as the geometrical center of the smallest rectangle that could be drawn around the worm) and recorded its $x$ and $y$ coordinates with a sampling rate of $2 \mathrm{~s}^{-1}$. When a worm neared the edge of the field of view, the tracking system automatically re-centered the worm by moving the stage and recorded the distance that the stage was moved. We reduced the variation in sampling rate as a consequence of the small differences in the time it took to re-center the worm and the need to take data only when the stage was stationary by developing a simultaneous localization and tracking method for a worm tracking system (Kowalski et al., 2014). The spatiotemporal track of each worm was reconstructed from the record of centroid locations and camera displacements. The instantaneous speed and trajectory were computed using the displacement of the centroid in successive samples. The tracking system recorded the worm's position, speed, distance from the center of the plate and from the starting point, estimated nicotine concentration in the surroundings the worm, and trajectory at 0.5 -s intervals. Individual worms moved away from their starting location, leaving complex tracks. Video recordings were carried out at room temperature $\left(22^{\circ} \mathrm{C}\right)$. Five independent experiments were performed per dosage group.

All the experimental procedures presented in this paper were in compliance with the European Communities Council Directive of 24 November 1986 (86/609/EEC).

\subsection{Data analysis and statistical analysis}

The measurements from experiments were pooled for each treatment variant and the median values were calculated. The data were not normally distributed, as determined by the Shapiro-Wilk $W$-test and Kolmogorov-Smirnov \& Lilliefors method. Due to this, the Kruskal-Wallis tests followed by Dunn's multiple comparison post-hoc tests were performed. Statistical significance was considered at $p<0.05$. The calculations and graphs were done by using Statistica software (StatSoft, Inc., Tulsa, Oklahoma, USA). 
bioRxiv preprint doi: https://doi.org/10.1101/099952; this version posted January 12,2017 . The copyright holder for this preprint (which was not certified by peer review) is the author/funder, who has granted bioRxiv a license to display the preprint in perpetuity. It is made available under aCC-BY-ND 4.0 International license.

461 Worm body size data are presented as mean \pm S.D. of at least 8 independent 462 experiments. One-way ANOVA followed by Scheffe's test were performed to determine 463 statistical differences between groups with the aid of Statistica (StatSoft software). 464 Significance was set at $p<0.05$.

465 


\section{Acknowledgments}

468 We would like to thank the Caenorhabditis Genetics Center, which is funded by the

469 NIH National Center for Research Resources (NCRR), for supplying the nematode strain used

470 in this research. We thank also Sylwia Ufnalska, an author's editor, for help in improving the

471 manuscript. This study was supported by funds from the Department of Cell Biology, Faculty

472 of Biology, Adam Mickiewicz University (Poznań, Poland).

473

474 


\section{References}

476 Altun, Z.F., Hall, D.H. 2016. Handbook of $C$. elegans anatomy. In WormAtlas.

477

478

479

480

481

482

483

484

485

486

487

488

489

490

491

492

493

494

495

496

497

498

499

500

501

502

503

504

505

506

507 http://www.wormatlas.org/hermaphrodite/hermaphroditehomepage.htm

Bargmann, C.I., 2006. Chemosensation in C. elegans, in: Community, T.C.e.R. (Ed.) WormBook. WormBook.

Benowitz, N.L., 2010. Nicotine addiction. N Engl J Med 362(24), 2295-2303.

Benowitz, N.L., Hukkanen, J., Jacob, P., 3rd, 2009. Nicotine chemistry, metabolism, kinetics and biomarkers. Handb Exp Pharmacol 192(192), 29-60.

Brenner, S., 1974. The genetics of Caenorhabditis elegans. Genetics 77(1), 71-94.

Chase, D.L., Koelle, M.R., 2007. Biogenic amine neurotransmitters in C. elegans, in: Community, T.C.e.R. (Ed.) Wormbook.

Crank, J., 1975. The mathematics of diffusion. Oxford: Clarenden.

de Bono, M., Bargmann, C.I., 1998. Natural variation in a neuropeptide Y receptor homolog modifies social behavior and food response in C. elegans. Cell 94(5), 679-689.

Feng, Z., Li, W., Ward, A., Piggott, B.J., Larkspur, E.R., Sternberg, P.W., Xu, X.Z., 2006. A C. elegans model of nicotine-dependent behavior: regulation by TRP-family channels. Cell 127(3), 621-633.

Filozof, C., Fernandez Pinilla, M.C., Fernandez-Cruz, A., 2004. Smoking cessation and weight gain. Obes Rev 5(2), 95-103.

Grana, R., Benowitz, N., Glantz, S.A., 2014. E-cigarettes: a scientific review. Circulation 129(19), 1972-1986.

Gray, J.M., Hill, J.J., Bargmann, C.I., 2005. A circuit for navigation in Caenorhabditis elegans. Proc Natl Acad Sci U S A 102(9), 3184-3191.

Iino, Y., Yoshida, K., 2009. Parallel use of two behavioral mechanisms for chemotaxis in Caenorhabditis elegans. J Neurosci 29(17), 5370-5380.

Jo, Y.H., Talmage, D.A., Role, L.W., 2002. Nicotinic receptor-mediated effects on appetite and food intake. J Neurobiol 53(4), 618-632.

Kowalski, M., Kaczmarek, P., Kabaciński, R., Matuszczak, M., Tranbowicz, K., Sobkowiak, R., 2014. A simultaneous localization and tracking method for a worm tracking system. International Journal of Applied Mathematics and Computer Science 24(3), 599-609.

Liu, R.H., Mizuta, M., Matsukura, S., 2004. The expression and functional role of nicotinic acetylcholine receptors in rat adipocytes. J Pharmacol Exp Ther 310(1), 52-58. 
508 Matsuura, T., Miura, H., Nishino, A., 2013. Inhibition of gustatory plasticity due to acute nicotine exposure in the nematode Caenorhabditis elegans. Neurosci Res 77(3), 155-161.

Matta, S.G., Balfour, D.J., Benowitz, N.L., Boyd, R.T., Buccafusco, J.J., Caggiula, A.R., Craig, C.R., Collins, A.C., Damaj, M.I., Donny, E.C., Gardiner, P.S., Grady, S.R., Heberlein, U., Leonard, S.S., Levin, E.D., Lukas, R.J., Markou, A., Marks, M.J., McCallum, S.E., Parameswaran, N., Perkins, K.A., Picciotto, M.R., Quik, M., Rose, J.E., Rothenfluh, A., Schafer, W.R., Stolerman, I.P., Tyndale, R.F., Wehner, J.M., Zirger, J.M., 2007. Guidelines on nicotine dose selection for in vivo research. Psychopharmacology (Berl) 190(3), 269-319.

McKay, J.P., Raizen, D.M., Gottschalk, A., Schafer, W.R., Avery, L., 2004. eat-2 and eat18 Are Required for Nicotinic Neurotransmission in the Caenorhabditis elegans Pharynx. Genetics 166(1), 161-169.

Mineur, Y.S., Abizaid, A., Rao, Y., Salas, R., DiLeone, R.J., Gundisch, D., Diano, S., De intake through activation of POMC neurons. Science 332(6035), 1330-1332.

Moore, B.T., Jordan, J.M., Baugh, L.R., 2013. WormSizer: high-throughput analysis of nematode size and shape. PLoS ONE 8(2), e57142.

Nichter, M., Vuckovic, N., Tesler, L., Adrian, S., Ritenbaugh, C., 2004. Smoking as a weight-control strategy among adolescent girls and young women: a reconsideration. Med Anthropol Q 18(3), 305-324.

Picciotto, M.R., Kenny, P.J., 2013. Molecular mechanisms underlying behaviors related to nicotine addiction. Cold Spring Harb Perspect Med 3(1), a012112.

Pierce-Shimomura, J.T., Morse, T.M., Lockery, S.R., 1999. The fundamental role of pirouettes in Caenorhabditis elegans chemotaxis. J Neurosci 19(21), 9557-9569.

Ramot, D., Johnson, B.E., Berry, T.L., Jr., Carnell, L., Goodman, M.B., 2008. The Parallel Worm Tracker: a platform for measuring average speed and drug-induced paralysis in nematodes. PLoS ONE 3(5), e2208.

Rose, J.K., Miller, M.K., Crane, S.A., Hope, K.A., Pittman, P.G., 2013. Parental and larval exposure to nicotine modulate spontaneous activity as well as cholinergic and GABA receptor expression in adult C. elegans. Neurotoxicol Teratol. a serotonergic pathway. Neuron 26(3), 619-631. 
541 Schuller, H.M., 2009. Is cancer triggered by altered signaling of nicotinic acetylcholine 542 receptors? Nat Rev Cancer 9(3), 195-205.

543 Sellings, L., Pereira, S., Qian, C., Dixon-McDougall, T., Nowak, C., Zhao, B., Tyndale,

544 R.F., van der Kooy, D., 2013. Nicotine-motivated behavior in Caenorhabditis elegans

545 requires the nicotinic acetylcholine receptor subunits acr-5 and acr-15. Eur J Neurosci.

546 Sobkowiak, R., Kowalski, M., Lesicki, A., 2011. Concentration- and time-dependent 547 behavioral changes in Caenorhabditis elegans after exposure to nicotine. Pharmacol $548 \quad$ Biochem Behav 99, 365-370.

549 Sobkowiak, R., Lesicki, A., 2011. Komórkowe szlaki sygnalizacyjne aktywowane przez 550 nikotynę. [Cell signaling pathways activated by nicotine]. Post Biol. Kom 38(4), 581-596.

551 Sobkowiak, R., Lesicki, A., 2013. Wchłanianie, przemiany metaboliczne i wydalanie 552 nikotyny u czlowieka. [Absorption, metabolism and excretion of nicotine in humans]. Post 553 Biochem 59(1), 33-44.

554 Sobkowiak, R., Zieleziński, A., Wojciech M Karlowski, W.M., Lesicki, A., 2017. Nicotine 555 affects protein complex rearrangement in Caenorhabditis elegans cells. Drug Chem 556 Toxicol, DOI: 10.1080/01480545.2016.1264411

557 Stiernagle, T., 2006. Maintenance of C. elegans, in: Community, T.C.e.R. (Ed.) WormBook. 558 WormBook.

559 Tsunozaki, M., Chalasani, S.H., Bargmann, C.I., 2008. A behavioral switch: cGMP and 560 PKC signaling in olfactory neurons reverses odor preference in C. elegans. Neuron 59(6), $561959-971$.

562 Waggoner, L.E., Dickinson, K.A., Poole, D.S., Tabuse, Y., Miwa, J., Schafer, W.R., 2000.

563 Long-term nicotine adaptation in Caenorhabditis elegans involves PKC-dependent 564 changes in nicotinic receptor abundance. J Neurosci 20(23), 8802-8811.

565 [WHO] World Health Organization, 2012. WHO global report: mortality attributable to 566 tobacco.

567 http://www.who.int/tobacco/publications/surveillance/rep_mortality_attributable/en/.

568 Wolf, F.W., Heberlein, U., 2003. Invertebrate models of drug abuse. J Neurobiol 54(1), 161$569 \quad 178$.

570 
571 Figure 1. Outline of experimental procedures. (A) Incubation. Caenorhabditis elegans were

572 grown for 5 days at $22^{\circ} \mathrm{C}$ on ENG medium plates spread with E. coli. (B) Long-term nicotine

573 adaptation. Adult worms were transferred to NGM plates seeded with food, by transferring of

574 chunks of the medium with nematodes onto control plates ( $0 \mathrm{mM}$ nicotine) and adaptation

575 plates containing $0.01 \mathrm{mM}$ nicotine (low nicotine concentration) or $1 \mathrm{mM}$ nicotine (high

576 nicotine concentration). All adult worms after $3 \mathrm{~h}$ were removed and only the laid eggs were

577 left. Incubation at $22^{\circ} \mathrm{C}$ for $55 \mathrm{~h}$. (C) Transfer of single adult nematodes onto gradient assay

578 plates. Duration of the experiment: $3600 \mathrm{~s}$. White plate $=$ no food; uniform gray plate $=$

579 presence of food; peak at the plate center = application point of nicotine at the beginning of

580 the experiment; no peak at the plate center = control plates (with water instead of nicotine).

581 (D) Data analysis of used behavioral endpoints measured in the tests.

582

583 Figure 2. (A) Configuration of the plate for nicotine gradient assay of Caenorhabditis 584 elegans. One worm was placed in a 1- $\mu \mathrm{L}$ drop of water and thus trapped for several dozen 585 seconds in the start position of a Petri dish containing $5.8 \mathrm{~mL}$ of NGM medium. Immediately 586 after the worm was released, nicotine was spotted into the center of the plate (peak). Water 587 was applied instead of nicotine in control variants. (B) Theoretical distribution of nicotine 588 concentration depending on the distance ( $r=$ radius) from the peak. The gradient was formed 589 by placing $1 \mu \mathrm{L}$ of $580 \mathrm{mM}(-)$-nicotine at the center of the plate at the beginning of 590 experiment. Nicotine appears to move smoothly and systematically from high-concentration 591 areas to low-concentration areas, following Fick's laws. Concentration estimates were made 592 as described in section 2.4 (see the equation)

594 Figure 3. Differences in body size of Caenorhabditis elegans after 55 h of growth in control 595 conditions $(0 \mathrm{mM}$ nicotine $)$ and in the presence of $0.01 \mathrm{mM}$ nicotine and $1 \mathrm{mM}$ nicotine $596 \quad(* p<0.05$, Scheffe test, $N=96$ at least).

597

598 Figure 4. Average values of factors describing the behavior of Caenorhabditis elegans: (A) 599 speed of movement; (B) distance from the start position; (C) distance from the peak; (d) 600 preferred nicotine concentration; "ns" denoted no statistically significant differences between 601 experimental conditions. Other groups were statistically significantly different from each 602 other $(p<0.05$, Kruskal-Wallis ANOVA by ranks test, data pooled from 5 independent 603 experiments, $N=14370$ ). 
604 Figure 5. Locomotor activity (centroid speed) of Caenorhabditis elegans in the tested 605 experimental variants. The data are medians and 25th and 75th percentiles of 5 pooled 606 experiments. F-/F+ = absence/presence of food, respectively; $\mathrm{n}=$ no nicotine gradient; $\mathrm{N}=$ 607 presence of nicotine gradient; $\mathrm{A} 0=$ naïve worms, which never had contact with nicotine 608 before; $\mathrm{A} 0.01=$ worms adapted to $0.01 \mathrm{mM}$ nicotine; $\mathrm{A} 1=$ worms adapted to $1 \mathrm{mM}$ nicotine.

609

610 Figure 6. Distance from the start position of Caenorhabditis elegans in the tested 611 experimental variants. The data are medians and 25th and 75th percentiles of 5 pooled 612 experiments. F-/F+ = absence/presence of food, respectively; $\mathrm{n}=$ no nicotine gradient; $\mathrm{N}=$ 613 presence of nicotine gradient; $\mathrm{A} 0=$ naïve worms, which never had contact with nicotine 614 before; $\mathrm{A} 0.01=$ worms adapted to $0.01 \mathrm{mM}$ nicotine; $\mathrm{A} 1=$ worms adapted to $1 \mathrm{mM}$ nicotine.

616 Figure 7. Time-dependent changes in distance from the peak of Caenorhabditis elegans in 617 the tested experimental variants. The data are medians and 25 th and 75 th percentiles of 5 618 pooled experiments. $\mathrm{F}-/ \mathrm{F}+=$ absence/presence of food, respectively; $\mathrm{n}=$ no nicotine gradient; $619 \mathrm{~N}=$ presence of nicotine gradient; $\mathrm{A} 0$ = naïve worms, which never had contact with nicotine 620 before; $\mathrm{A} 0.01=$ worms adapted to $0.01 \mathrm{mM}$ nicotine; $\mathrm{A} 1=$ worms adapted to $1 \mathrm{mM}$ nicotine.

621

622 Figure 8. Time-dependent changes in nicotine concentration preferred by Caenorhabditis 623 elegans in the tested experimental variants. The data are medians and 25th and 75th 624 percentiles of 5 experiments. F-/F+ = absence/presence of food, respectively; $\mathrm{n}=$ no nicotine 625 gradient; $\mathrm{N}=$ presence of nicotine gradient; $\mathrm{A} 0=$ naïve worms, which never had contact with 626 nicotine before; $\mathrm{A} 0.01=$ worms adapted to $0.01 \mathrm{mM}$ nicotine; $\mathrm{A} 1=$ worms adapted to $1 \mathrm{mM}$ 627 nicotine. 
(a) C. elegans N2 culture, ENG plate

5 days

(b) Adaptation to (-)- nicotine

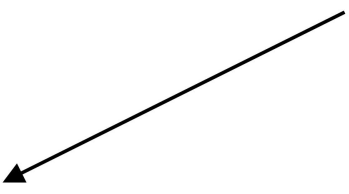

$55 \mathrm{~h}$

(c) Gradient assay $3600 \mathrm{~s}$
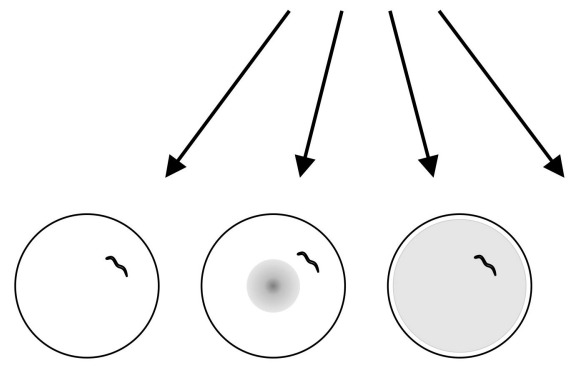

$0 \mathrm{mM}$ nicotine (no gradient)
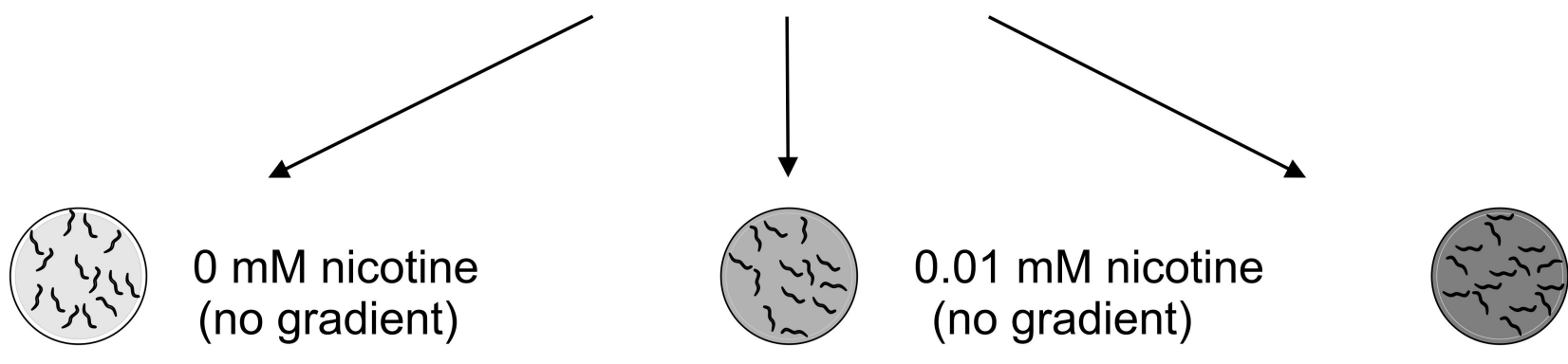

$1 \mathrm{mM}$ nicotine (no gradient)

\section{Food (F) - E. coli OP50}

$(-)$
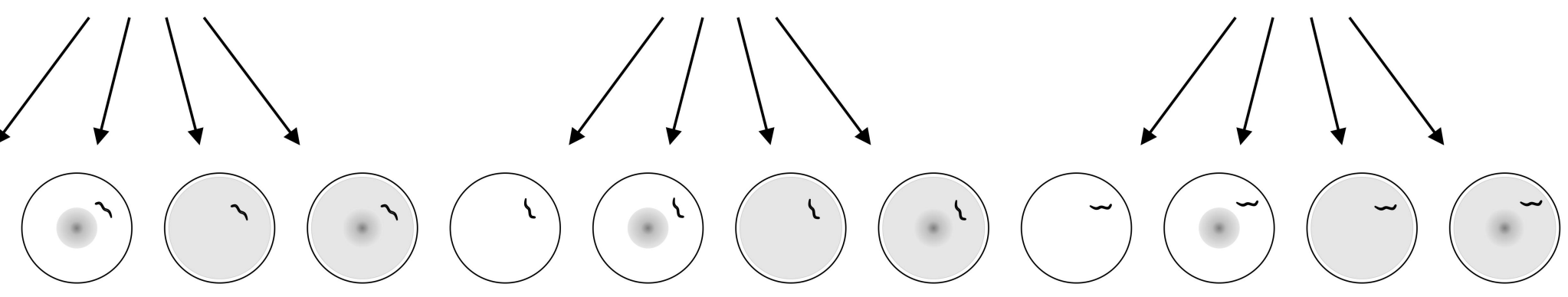

\section{Central $1 \mu \mathrm{L}$ spot}

of $580 \mathrm{mM}$ nicotine $(\mathrm{N})$

or water $(\mathrm{n})$ :

$\mathrm{n}$

$(-)$

$(+)$

$(+)$

$(-)$

$(-)$

$(+)$

$(+)$

$(-)$

$(-)$

(+)

$(+)$

Nicotine adaptation $(A): \quad 0$

N

N

n

N

n

N

$\mathrm{n}$

N

n

N

0

$0 \quad 0.01$

0.01

0.01

0.01

$\mathrm{F}+\mathrm{nA0} 0.01$

1

1

1

$1 \mathrm{mM}$

Unique description: F-nA0

$\mathrm{F}+\mathrm{nA0}$

F-nA0.01

F-nA1

$\mathrm{F}+\mathrm{nA} 1$

F-NAO

$\mathrm{F}+\mathrm{NAO}$

F-NA0.01

$\mathrm{F}+\mathrm{NA} 0.01$

F-NA1

F+NA1

(d) Data analysis

speed of movement, preferred nicotine concentration, distance from central spot, and starting point

Figure 1. Outline of experimental procedures. (A) Incubation. Caenorhabditis elegans were grown for 5 days at $22^{\circ} \mathrm{C}$ on $\mathrm{ENG}$ medium plates spread with E. coli. (B) Long-term nicotine adaptation. Adult worms were transferred to NGM plates seeded with food, by transferring of chunks of the medium with nematodes onto control plates ( $0 \mathrm{mM}$ nicotine) and adaptation plates containing $0.01 \mathrm{mM}$ nicotine (low nicotine concentration) or 1 $\mathrm{mM}$ nicotine (high nicotine concentration). All adult worms after $3 \mathrm{~h}$ were removed and only the laid eggs were left. Incubation at $22^{\circ} \mathrm{C}$ for $55 \mathrm{~h}$. (C) Transfer of single adult nematodes onto gradient assay plates. Duration of the experiment: $3600 \mathrm{~s}$. White plate $=$ no food; uniform gray plate $=$ presence of food; peak at the plate center = application point of nicotine at the beginning of the experiment; no peak at the plate center $=$ control plates (with water instead of nicotine). (D) Data analysis of used behavioral endpoints measured in the tests. 


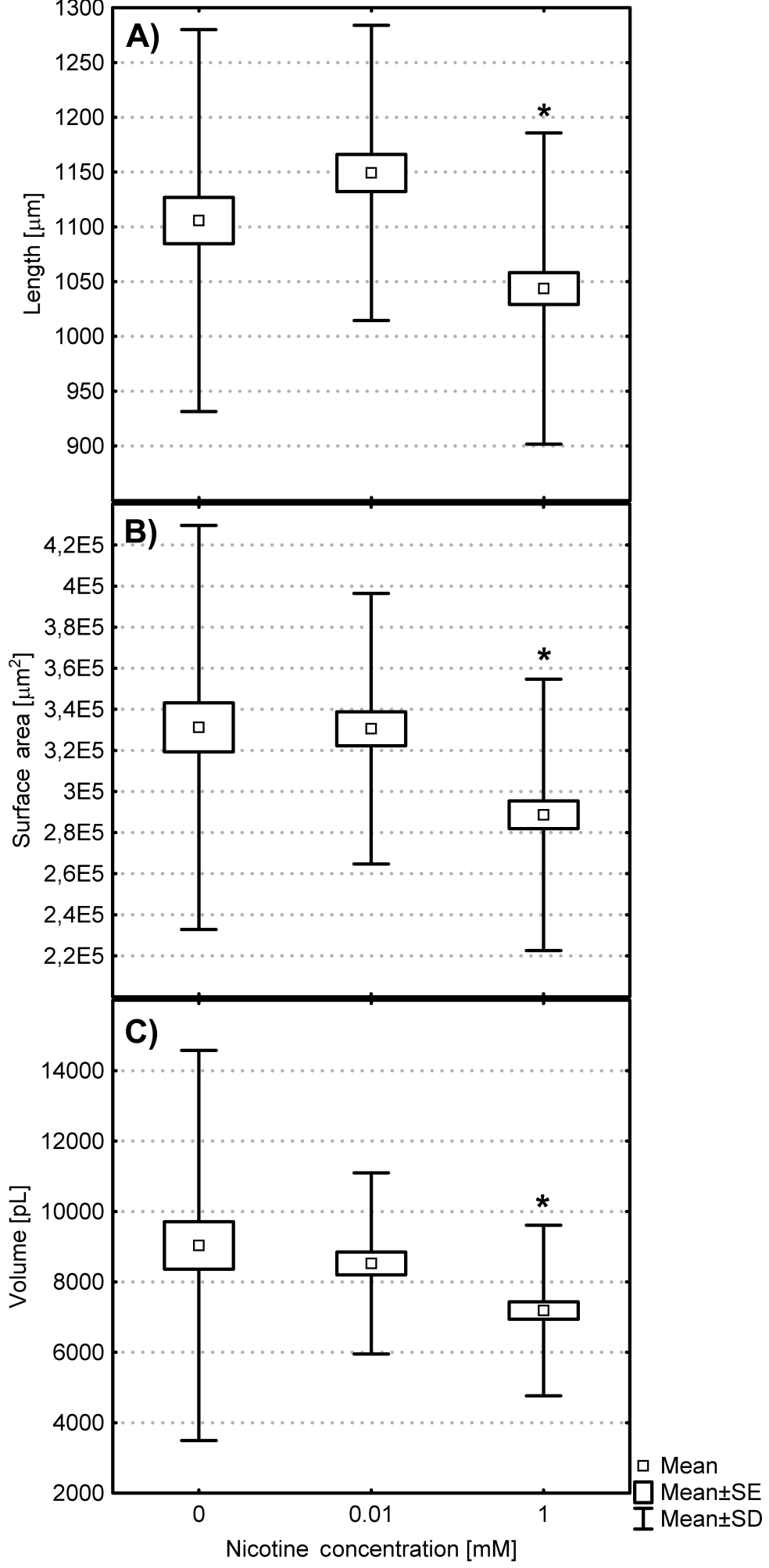

Figure 3. Differences in body size of Caenorhabditis elegans after $55 \mathrm{~h}$ of growth in control conditions ( $0 \mathrm{mM}$ nicotine $)$ and in the presence of $0.01 \mathrm{mM}$ nicotine and $1 \mathrm{mM}$ nicotine ( ${ }^{*} p<0.05$, Scheffe test, $N=96$ at least). 


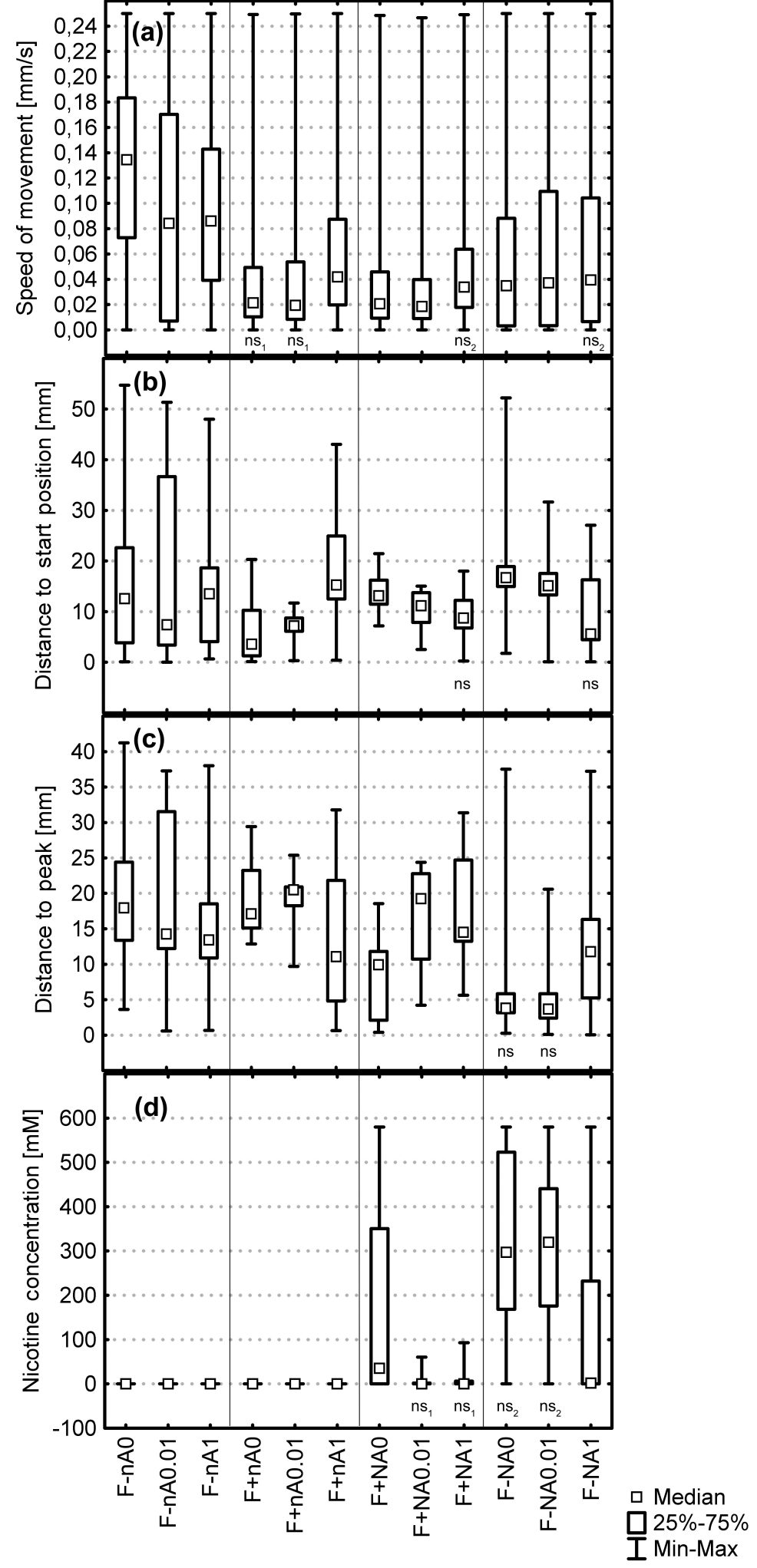

Experimental condition

Figure 4. Average values of factors describing the behavior of Caenorhabditis elegans: (A) speed of movement; (B) distance from the start position; (C) distance from the peak; (d) preferred nicotine concentration; "ns" denoted no statistically significant differences between experimental conditions. Other groups were statistically significantly different from each other $(p<0.05$, Kruskal-Wallis ANOVA by ranks test, data pooled from 5 independent experiments, $\mathrm{N}=14370$ ). 

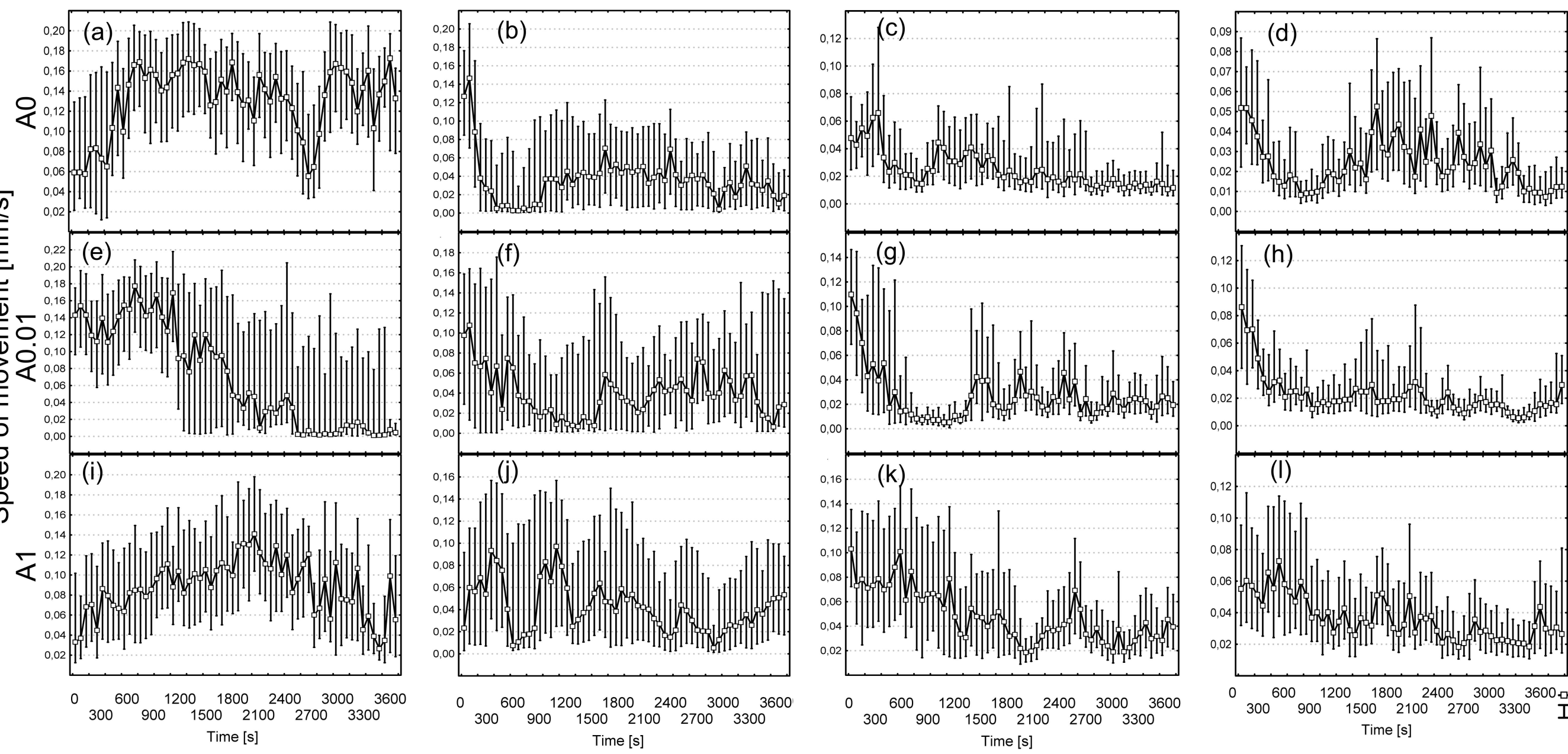

(h)

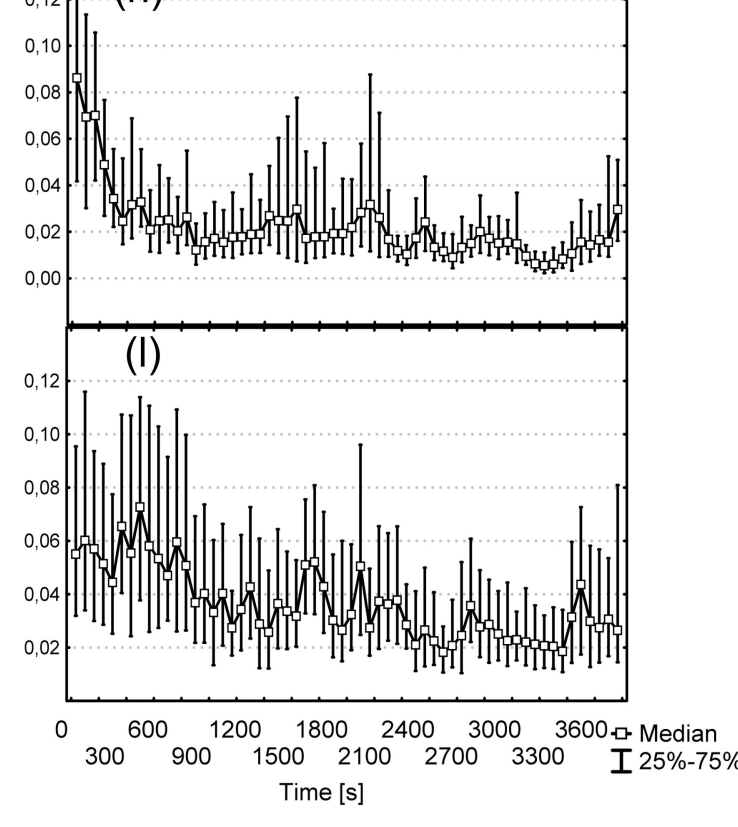

Figure 5. Locomotor activity (centroid speed) of Caenorhabditis elegans in the tested experimental variants. The data are medians and 25th and 75th percentiles of 5 pooled experiments. $\mathrm{F}-/ \mathrm{F}+=$ absence/presence of food, respectively; $n=$ no nicotine gradient; $\mathrm{N}=$ presence of nicotine gradient; $A 0=$ naïve worms, which never had contact with nicotine before; $A 0.01=$ worms adapted to 0.01 mM nicotine; $A 1=$ worms adapted to $1 \mathrm{mM}$ nicotine. 

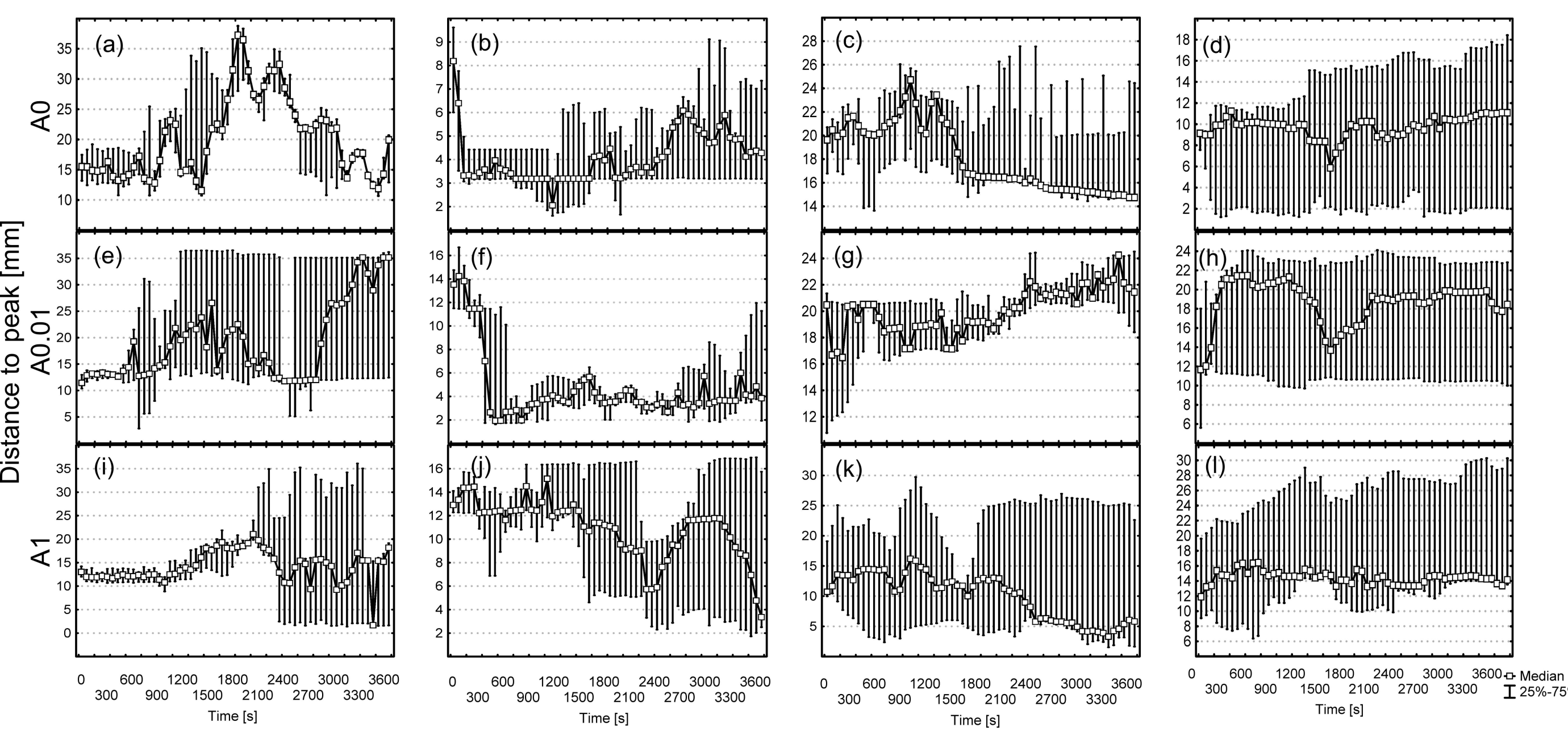

Figure 7. Time-dependent changes in distance from the peak of Caenorhabditis elegans in the tested experimental variants. The data are medians and 25th and 75th percentiles of 5 pooled experiments. $\mathrm{F}-/ \mathrm{F}+=$ absence/presence of food, respectively; $\mathrm{n}=\mathrm{no}$ nicotine gradient; $\mathrm{N}=$ presence of nicotine gradient; $A O=$ naïve worms, which never had contact with nicotine before; $A 0.01=$ worms adapted to 0.01 mM nicotine; $\mathrm{A} 1=$ worms adapted to $1 \mathrm{mM}$ nicotine 

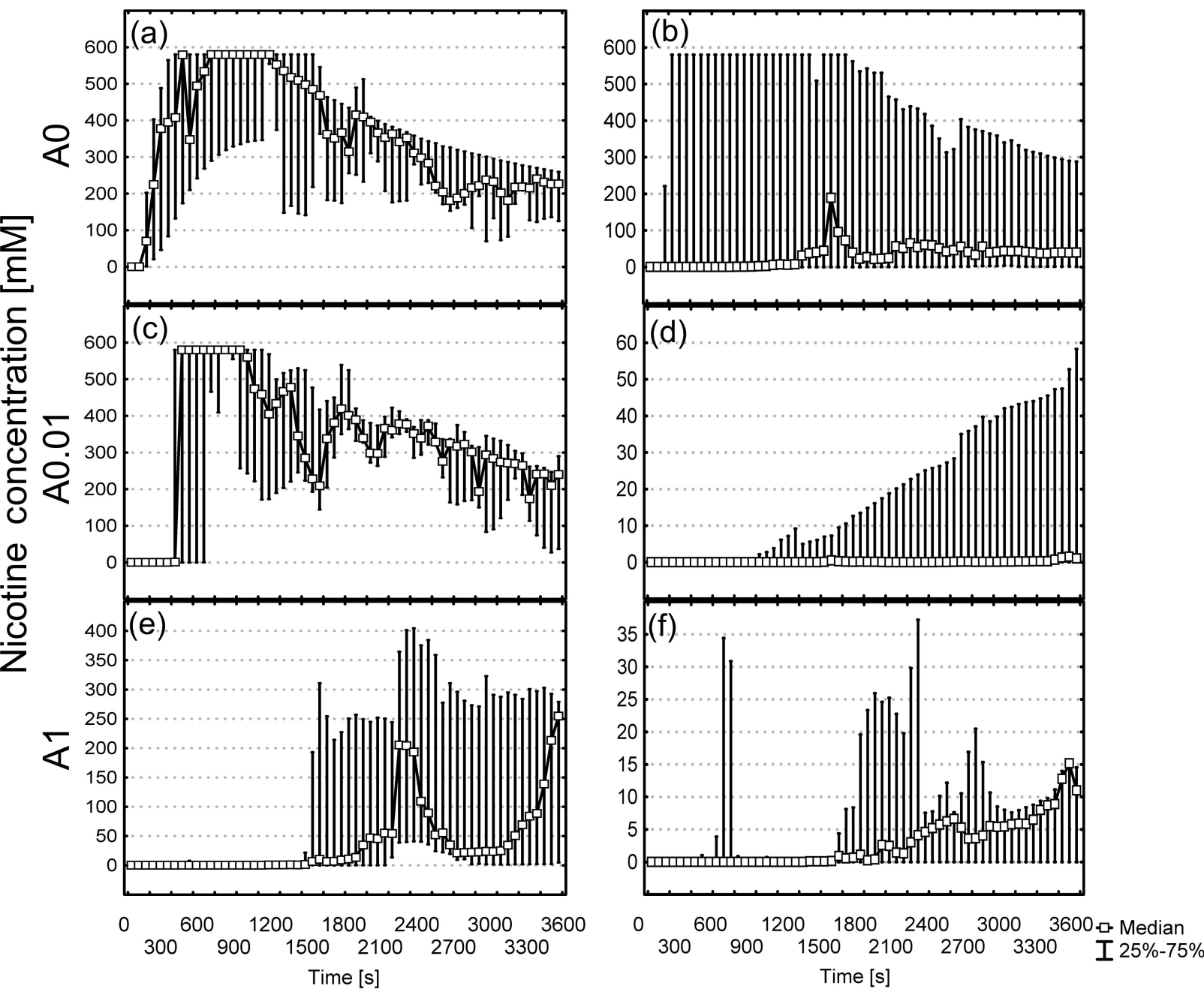

Figure 8. Time-dependent changes in nicotine concentration preferred by Caenorhabditis elegans in the tested experimental variants. The data are medians and 25th and 75 th percentiles of 5 experiments. $F-/ F+=$ absence/presence of food, respectively; $\mathrm{n}=$ no nicotine gradient; $\mathrm{N}=$ presence of nicotine gradient; $\mathrm{A} 0=$ naïve worms, which never had contact with nicotine before; $A 0.01=$ worms adapted to $0.01 \mathrm{mM}$ nicotine; $\mathrm{A} 1=$ worms adapted to $1 \mathrm{mM}$ nicotine. 